NASA Contractor Report 182103

ICASE Report No. 90-62

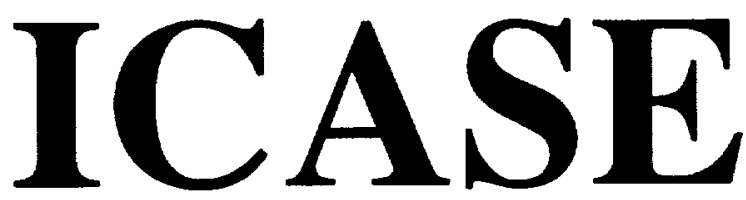

BOUNDARY-LAYER RECEPTIVITY DUE TO A WALL SUCTION AND CONTROL OF TOLLMIENSCHLICHTING WAVES

R. J. Bodonyi
P. W. Duck

Contract No. NAS1-18605

September 1990

Institute for Computer Applications in Science and Engineering NASA Langley Research Center

Hampton, Virginia 23665-5225

Operated by the Universities Space Research Association

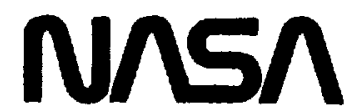

National Aeronautics and

Space Administration

Langley Research Center

Harmplon, Virginia 23665-5225

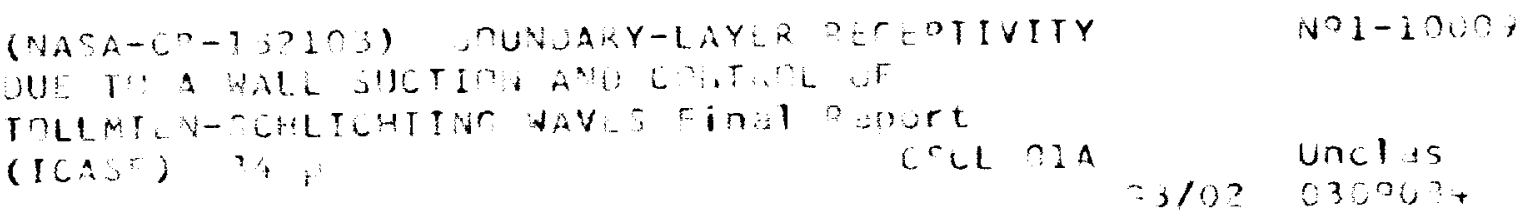





\title{
Boundary-Layer Receptivity Due to a Wall Suction and Control of Tollmien-Schlichting Waves
}

\author{
R. J. Bodonyi \\ Department of Aeronautical \& Astronautical Engineering \\ The Ohio State University \\ 2036 Neil Ave. Mall \\ Columbus, Ohio 43210 \\ and \\ P. W. Duck ${ }^{1}$ \\ Department of Mathematics \\ University of Manchester \\ Manchester M13-9PL \\ United Kingdom
}

\begin{abstract}
A numerical study of the generation of Tollmien-Schlichting waves due to the interaction between a small free-stream disturbance and a small localized suction slot on an otherwise flat surface has been carried out using finite-difference methods. The nonlinear steady flow is of the viscous-inviscid interactive type while the unsteady disturbed flow is assumed to be governed by the Navier-Stokes equations linearized about this flow. Numerical solutions illustrate the growth or decay of the T-S waves generated by the interaction between the freestream disturbance and the suction slot, depending on the value of the scaled Strouhal number. An important result of this receptivity problem is the numerical determination of the amplitude of the Tollmien-Schlichting waves and the demonstration of the possible active control of the growth of Tollmien-Schlichting waves.
\end{abstract}

\footnotetext{
${ }^{1}$ Research was supported by the National Aeronautics and Space Administration under NASA Contract No. NAS1-18605 while the author was in residence at the Institute for Computer Applications in Science and Engineering (ICASE), NASA Langley Research Center, Hampton, VA 23665.
} 


\section{Introduction}

With the development of more complicated flight vehicles the prediction and control of boundary-layer transition have become ever increasing areas of interest. Furthermore, the problem of boundarylayer receptivity due to free-stream acoustic disturbances has been of renewed interest recently since it is believed that this receptivity plays a key role in the early stages of the transition of a boundary-layer flow from a laminar to a turbulent state.

Early studies of the receptivity problem have been reviewed by Reshotkol and more recent studies include the work of Murdock ${ }^{2}$, Goldstein ${ }^{3}, 4,5$, Goldstein, Sockol and $\operatorname{Sanz}^{6}$, Goldstein, Leib and Cowley ${ }^{7}$, and Bodonyi, Welch, Duck and Tadjfar ${ }^{8}$. Here the role of small free-stream disturbances in the generation of TollmienSchlichting $(\mathrm{T}-\mathrm{S})$ waves have been examined in detail for a variety of problems indicating the important role that nonparallel flow effects have on the receptivity mechanisms. In particular, the studies by Goldstein and his co-workers and Bodonyi et al have shown the effects that small variations in surface geometry have on scattering weak unsteady free-stream disturbances into $\mathrm{T}$-S waves using tripledeck theory. Their results showed conclusively that relatively small surface variations which set up equally small pressure changes can produce an order one coupling between the $\mathrm{T}-\mathrm{S}$ waves and the imposed disturbance when these variations are sufficiently rapid that they occur on the scale of a T-S wave.

Bodonyi et al. 8 showed that for sufficiently small surface 
distortions such that the steady flow is governed by linear equations, the scaled disturbance amplitudes depend approximately linearly on the surface height. For larger values of the surface height parameter, wherein the basic flow is governed by the full nonlinear triple-deck equations, their results showed that there is an increasingly nonlinear enhancing effect on the disturbance amplitudes.

The concept of transition control has also been of renewed interest lately, with an emphasis on attempting to control linearized boundary-layer disturbances actively. Liepman, Brown and Nosenchuck 9 and Liepman and Nosenchuck ${ }^{10}$ have demonstrated experimentally that nearly complete cancellation of a $\mathrm{T}-\mathrm{S}$ wave excited by the periodic heating of flush-mounted elements can be achieved by using a second downstream element with a suitable phase shift. We demonstrate that such wave cancellations are also possible in this study by appropriately choosing the wall suction velocity.

Following the work of Bodonyi et al. 8 we take the steady nonlinear viscous-inviscid interactive solutions of the triple-deck kind for the basic steady motion of a localized suction slot on an otherwise flat surface as the basic flow. Additionally, we shall assume that the unsteady flow is governed by the Navier-stokes equations linearized about the nonlinear steady state as in Smithll. 


\section{Problem Formulation}

We wish to study the interaction between an unsteady freestream and a localized suction slot on a flat plate for an incompressible two-dimensional viscous flow. Thus following Bodonyi et al. 8 we take the upstream motion to consist of a uniform flow with velocity $U_{\infty}^{*}$ plus a small harmonic perturbation of frequency $\Omega$ and constant amplitude $u_{\infty}^{*} \ll U_{\infty}^{*}$, so that the unsteady motion can be analyzed as a Iinear perturbation of the uniform steady flow, $U_{\infty}^{*}$ i.e., $U_{\infty}^{*}(1+$ $\left.u_{\infty}^{*} / U_{\infty}^{*} e^{-i \Omega t^{*}}\right)$.

Consider a Cartesian coordinate system $\left(x^{*}, Y^{*}\right)$ with $x^{*}$ tangent to and $y^{*}$ normal to the flat plate with the origin taken at the leading edge. Further, define the Reynolds number $\operatorname{Re}=U_{\infty}^{*} L^{*} / \nu$, where $L^{*}$ is the distance of the surface perturbation from the leading edge of the flat plate and $\nu$ is the kinematic viscosity of the fluid. For convenience, we introduce the small parameter $\varepsilon=R e^{-1 / 8}$ and consider solutions of the Navier-Stokes equations when $\operatorname{Re} \gg 1$. Specifically, we wish to consider the problem of flow over a small slot of length $O\left(L^{*} \varepsilon^{3}\right)$ positioned at a distance $L^{*}$ from the leading edge. The interaction region is shown schematically in Figure 1.

Thus we take

$$
\begin{aligned}
& \hat{U}(x, y, t)=U_{0}(x, y)+\delta \tilde{u}(x, y, t), \\
& \hat{v}(x, y, t)=v_{0}(x, y)+\delta \tilde{v}(x, y, t), \\
& \hat{P}(x, y, t)=P_{0}(x, y)+\delta \tilde{p}(x, y, t),
\end{aligned}
$$

where the steady velocity components, $U_{0^{\prime}} \mathrm{V}_{0^{\prime}}$ and pressure, $\mathrm{P}_{\circ}$, are normalized by $U_{\infty}^{*}$ and $\rho U_{\infty}^{* 2}$, respectively and $\tilde{u}, \tilde{v}, \tilde{p}$ the unsteady velocity and pressure terms normalized by $u_{\infty}^{\star}$ and $\rho u_{\infty}^{\star} U_{\infty}^{*}$ 
respectively. Furthermore, we define

$$
x=\left(x^{*}-L^{*}\right) / L^{*}, y=y^{*} / L^{*}, \quad t=\Omega t^{*}, \quad \delta=u_{\infty}^{*} / U_{\infty}^{*} .
$$

Substituting (1) - (2) into the Navier-stokes equations and neglecting terms of $O\left(\delta^{2}\right)$, we obtain the linearized perturbation equations

$$
\begin{aligned}
& S \frac{\partial \tilde{u}}{\partial t}+U_{o} \frac{\partial \tilde{u}}{\partial x}+\tilde{u} \frac{\partial U_{o}}{\partial x}+v_{o} \frac{\partial \tilde{u}}{\partial y}+\tilde{v} \frac{\partial U_{o}}{\partial y}=-\frac{\partial \tilde{p}}{\partial x}+\frac{1}{R e}\left[\frac{\partial^{2} \tilde{u}}{\partial x^{2}}+\frac{\partial^{2} \tilde{u}}{\partial y^{2}}\right], \\
& S \frac{\partial \tilde{v}}{\partial t}+U_{o} \frac{\partial \tilde{v}}{\partial x}+\tilde{u} \frac{\partial v_{o}}{\partial x}+v_{o} \frac{\partial \tilde{v}}{\partial y}+\tilde{v} \frac{\partial v_{o}}{\partial y}=-\frac{\partial \dot{p}}{\partial y}+\frac{1}{R e}\left[\frac{\partial^{2} \tilde{v}}{\partial x^{2}}+\frac{\partial^{2} \tilde{v}}{\partial y^{2}}\right], \\
& \frac{\partial \bar{u}}{\partial x}+\frac{\partial \bar{v}}{\partial \bar{y}}=0,
\end{aligned}
$$

where

$$
S=\Omega L^{*} / U_{\infty}^{*}
$$

is the strouhal number.

Finally, we note that the physical interaction between the oncoming boundary layer, freestream disturbance, and slot is governed by a triple-deck structure, centered near the surface slot. The details of the structure as applied to this type of problem have been given by Goldstein ${ }^{5}$ and we, therefore, only summarize the relevant portions here. As is usually the case the viscous interaction problem essentially reduces to a study of the lower-deck equations. Thus the appropriately scaled variables in the lower deck for the steady flow are

$$
\begin{aligned}
& U_{\circ}(X, Y)=\varepsilon U(X, Y)+O\left(\varepsilon^{2}\right), \\
& V_{\circ}(X, Y)=\varepsilon^{3} V(X, Y)+O\left(\varepsilon^{4}\right), \\
& P_{\circ}(X, Y)=\varepsilon^{2} P(X)+O\left(\varepsilon^{3}\right),
\end{aligned}
$$

where 


$$
X=X / \varepsilon^{3}, \quad Y=Y / \varepsilon^{5} .
$$

$U, V$, and $P$ are found from the solution of the lower-deck equations:

$$
\begin{gathered}
\frac{\partial U}{\partial X}+\frac{\partial V}{\partial Y}=0, \\
U \frac{\partial U}{\partial X}+V \frac{\partial U}{\partial Y}=-\frac{\partial P}{\partial X}+\frac{\partial^{2} U}{\partial Y^{2}}
\end{gathered}
$$

subject to the boundary conditions

$$
\begin{aligned}
& U=0, V=V_{W} \text { for }|X|<X_{S I}, V=0 \text { for }|X|>X_{S I} \\
& (U, V, P) \rightarrow(Y, 0,0) \text { as }|X| \rightarrow \infty, \\
& U \rightarrow[Y+A(X)], Y \rightarrow \infty, \text { all } X,
\end{aligned}
$$

where we have assumed that a simple renormalization of the variables has been carried out in order to set the value of the wall shear of the oncoming, undisturbed boundary layer to unity.

Finally, the interaction condition for incompressible flow, given by the Cauchy Hilbert integral,

$$
P(X)=1 / \pi \int_{-\infty}^{\infty}(X-\xi)^{-1}(d A / d \xi) d \xi,
$$

is used to close the problem mathematically.

For the unsteady flow we introduce the following lower-deck variables

$$
\begin{aligned}
& \tilde{u}(x, y, t)=e^{-i t} u(X, Y)+o(\varepsilon), \\
& \tilde{v}(x, y, t)=\varepsilon^{2} e^{-i t} v(X, Y)+o\left(\varepsilon^{3}\right), \\
& \tilde{p}(x, y, t)=\varepsilon e^{-i t} p(X)+o\left(\varepsilon^{2}\right) .
\end{aligned}
$$

In these expressions we have utilized the fact that since the unsteady flow is governed by the linearized Navier-stokes equations we can seek solutions which have a harmonic time dependence. Substituting (4d) and (8) into (3a) - (3c) yields, to leading order in $\varepsilon$, 


$$
\begin{gathered}
-i \varepsilon{ }^{2} S u+U \frac{\partial u}{\partial X}+u \frac{\partial U}{\partial X}+v \frac{\partial u}{\partial Y}+v \frac{\partial U}{\partial Y}=-\frac{\partial p}{\partial X}+\frac{\partial^{2} u}{\partial Y^{2}}, \\
\frac{\partial u}{\partial X}+\frac{\partial v}{\partial Y}=0,
\end{gathered}
$$

As noted in the Introduction, our interest in this paper is in the relatively high frequency case where we choose $\Omega$ to be of the same order as the Tollmien-Schlichting wave frequency at and upstream of the lower branch of the neutral stability curve. For this reason we require that $\Omega=O\left(\varepsilon^{-2}\right)$ i.e., the strouhal number $S=O\left(\varepsilon^{-2}\right)$. Thus we define a scaled strouhal number $s_{0}$ such that

$$
S_{0}=\varepsilon^{2} S, \quad S_{0}=O(1) \text {. }
$$

Furthermore, in this case the stokes-layer thickness is found to be of $O(\varepsilon)$ also, and, therefore, it will be of $O\left(\varepsilon^{5}\right)$ in terms of the lower-deck scalings. Thus the stokes-layer thickness is of the same order as the lower-deck thickness. Upstream of the triple-deck region, where the mean flow changes on the scale of $x$, the unsteady flow in the boundary layer is given by the stokes solution, which can be written in terms of the lower-deck scalings, as

$$
\begin{aligned}
& u=1-\exp \left[i^{3 / 2} S_{0}^{1 / 2} Y\right], \\
& p=i x s_{\circ} .
\end{aligned}
$$

The lower-deck problem is completed by solving (9) using (10) subject to the no slip condition at the wall

$$
\mathrm{u}=\mathrm{v}=0 \text { on } \mathrm{Y}=0 \text {, }
$$

matching with the main-deck solution

$$
u \rightarrow 1+a(X), \quad Y \rightarrow \infty, \quad a l l X,
$$

and matching with the upstream stokes layer solution given by (11) for $x \rightarrow-\infty$. To mathematically close the boundary-value problem a 
relationship between the disturbance pressure and displacement thickness must be given. For reasons discussed by Bodonyi et al. 8 the Hilbert integral relation used by Goldstein ${ }^{5}$ is not appropriate. Thus an alternative method based on that used by Bodonyi \& Duck ${ }^{12}$ in treating three-dimensional interacting flows has been employed here (and in Bodonyi et al. ${ }^{8}$ ). In this approach, the relationship between the disturbance pressure, $p(X)$, and displacement thickness, $-a(X)$, is found through a numerical solution of the upper-deck equations as opposed to a Hilbert integral representation. Specifically, it can be shown that the appropriate boundary-value problem in the upperdeck for the disturbance pressure is given by

$$
\frac{\partial^{2} \hat{p}}{\partial X^{2}}+\frac{\partial^{2} \hat{p}}{\partial \hat{Y}^{2}}=0
$$

with boundary conditions

$$
\begin{gathered}
\frac{\partial \hat{p}}{\partial \hat{y}}(X, 0)=\frac{d^{2} a}{d x^{2}}, \\
\hat{p}(X, \hat{Y}) \rightarrow 0, \hat{Y} \rightarrow \infty, \text { all } X \\
\hat{p}(X, \hat{y})+i s x \rightarrow p(X) \text { as } \hat{y} \rightarrow 0 \\
\hat{p} \rightarrow 0 \text { as } X \rightarrow-\infty \\
\frac{\partial \hat{p}}{\partial X}-i k \hat{p} \rightarrow 0 \text { as } X \rightarrow \infty
\end{gathered}
$$

where we have written

$$
\mathrm{p}-\mathrm{p}_{\infty}=\varepsilon\left[\hat{\mathrm{p}}(\mathrm{X}, \hat{\mathrm{Y}})+i \mathrm{~s}_{\mathrm{o}} \mathrm{X}\right], \hat{\mathrm{y}}=\mathrm{y} / \varepsilon^{3} .
$$

Note that $(14 \mathrm{e})$ defines a radiation condition applied on the disturbance pressure at the downstream boundary to simulate the outward propagating pressure Tollmien-Schlichting disturbances there. The wave number, $k$, which depends on $s_{\circ}$, is found from the solution 
of the classical orr-Sommerfeld eigenvalue problem (e.g. Duck ${ }^{13}$ ). Alternatively, $k$ can be computed iteratively from the numerical computations, as discussed by Bodonyi et al. ${ }^{8}$. 
III. Numerical Method

\section{A. Steady-state solution}

First we consider the steady flow problem defined by equations (5) - (7). Numerical solutions have been found using a finitedifference procedure developed by Smith \& Bodonyil4. Briefly, the governing equations are replaced by difference representations for $\psi$, $\mathrm{U}=\psi_{Y^{\prime}} \tau=U_{Y^{\prime}}$, and $P$ with uniform steps in $X, Y$. The computational domain extends from $X=X_{1}(<0)$ to $X=X_{2}(>0)$ and from $Y=0$ to $Y=$ $Y_{\infty}$, with starting conditions $(6 b)$ specified, in effect, at $X=X_{1}$.

The nonlinear difference equations at a given streamwise location $X$ are solved to within a tolerance of $10^{-6}$ in absolute value by Newtonian iteration using Gaussian elimination and back substitution. The solution is then advanced to the next streamwise location and the process repeated until the entire domain is covered. Since the problem is interactive, multiple forward-marching sweeps are necessary until a tolerance of $10^{-5}$ between successive values obtained for $P(X)$ is satisfied for all $X$. At this point the solution is said to have converged in the global sense. The diagonally dominant nature of the finite-difference form of the interaction law (2.18) makes this multi-sweeping process both fast and stable. Whenever flow reversal occurs, i.e., $U<0$, windward differencing is used to represent $U_{X}$ in finite-difference form.

The numerical solution has been found for several values of the wall suction parameter, $V_{W}$, and slot length parameter. $\mathrm{X}_{\mathrm{Sl}}$. Representative distributions of the wall shear $r(X, 0)$, and pressure, $P(X)$, of the steady flow are given in Figure 2 for $V_{W}=-0.5$ and $x_{S l}$ 
$P(X)$, of the steady flow are given in Figure 2 for $V_{W}=-0.5$ and $X_{S l}$ $=0.50$.

With the steady solution known, we now proceed to consider the numerical solution of the complex unsteady linearized boundary-layer equations (9). In our approach, the governing equations were solved in the physical plane using a finite-difference method.

\section{B. Unsteady Solution}

The unsteady equations (9) were replaced by a system of difference equations of second-order accuracy to be consistent with the numerical method used for the steady flow problem. Since the governing equations are linear no iteration in the normal direction is necessary at a fixed streamwise location. A single sweep across the boundary-layer region was sufficient to determine the solution there. Thus one complete sweep of the computational domain could be accomplished quickly. Multiple sweeps of the entire domain are still necessary to obtain the global solution, however, due to the elliptic nature of equations (13) - (14).

For numerical convenience we subtract out the stokes solution. Thus consider the following change of variables

$$
\begin{aligned}
& u(X, Y)=1-\exp \left[i^{3 / 2} S_{0}^{1} / 2 Y\right]+u_{0}(X, Y), \\
& v(X, Y)=v_{0}(X, Y), \frac{d p}{d X}-i s_{0}=\frac{d p_{0}}{d X} .
\end{aligned}
$$

Then the disturbance equations can be written as

$$
\frac{\partial u_{0}}{\partial X}+\frac{\partial v_{O}}{\partial Y}=0 \text {, }
$$




$$
\begin{aligned}
\left(\frac{\partial U}{\partial X}-\right. & \left.i S_{0}\right) u_{0}+U \frac{\partial u_{0}}{\partial X}+\frac{\partial U}{\partial Y} v_{0}+v \frac{\partial u_{O}}{\partial Y}+\frac{d p_{O}}{d X}-\frac{\partial^{2} u_{0}}{\partial Y^{2}}= \\
& -\frac{\partial U}{\partial X}+\left[\frac{\partial U}{\partial X}+i^{3 / 2} S_{0}^{\frac{1}{2}} V\right] \cdot \exp \left[i^{3 / 2} S_{0}^{\frac{1}{2}} Y\right],
\end{aligned}
$$

with boundary conditions

$$
\begin{aligned}
& \mathrm{u}_{0}=\mathrm{v}_{0}=0 \text { on } \mathrm{Y}=0 \text {, all } \mathrm{X}, \\
& \mathrm{u}_{0} \rightarrow 0 \text { as } \mathrm{X} \rightarrow-\infty, \mathrm{all} \mathrm{Y}, \\
& \mathrm{u}_{0}-\mathrm{a}(\mathrm{X})+\exp \left[\mathrm{i}^{3 / 2} \mathrm{~S}_{0}^{1 / 2} \mathrm{Y}\right] \text { as } \mathrm{Y} \rightarrow \infty, \text { all } \mathrm{X} .
\end{aligned}
$$

Given a guess or an update for $u_{0^{\prime}} v_{0^{\prime}} p_{0}(X)$ and $\hat{p}(X, \hat{Y})$ everywhere, equations (17) - (18) are marched forward in $\mathrm{X}$, while simultaneously equations (13) - (14) are solved along a line of varying $\hat{y}$. This then determines the complex-valued functions $u_{0}$ ' $v_{0}$, $p$ and $\hat{p}$ (and hence $a(X)$ ) at a given streamwise location $x$. Sweeping through all $\mathrm{x}$ stations constitutes one global iteration. Convergence is finally attained when a global convergence test on $a(X)$ is satisfied.

The main features of the numerical scheme are the following. Two and three-point differencing in $Y$ is used for equations (17a) and (17b), respectively, with equation (18c) applied at $Y=Y_{\infty}$. Threepoint central differencing is used to approximate (13) in both dimensions, while condition $(14 b)$ is applied at $\hat{y}=\hat{Y}_{\infty}$. Equation (14a) is approximated by one-sided differencing in $\hat{y}$ and a secondorder scheme for $\mathrm{X}$ derivatives. Finally, the radiation condition is applied in the following form to estimate the disturbance pressure at the downstream boundary

$$
\hat{p}\left(x_{\max }, \hat{y}\right)=\left[\hat{p}\left(x_{\max }-2 \Delta x, \hat{y}\right)-4 \hat{p}\left(x_{\max }-\Delta x, \hat{y}\right)\right] /[2 i k \Delta x-3] .
$$


The value for $k$ is either prescribed as discussed earlier, or by estimating its value from the relation $(\partial \hat{p} / \partial X) / i \hat{p}$ from values of $x$ reasonably far downstream, and then feeding this value back into the numerical computations. Numerically, the results indicate only slight differences in estimating $k$ in these two ways. This perhaps is not too surprising since the viscous-inviscid interaction is a local phenomenon and the behavior far downstream should approach that of the classical stability theory.

Supposing we have $n$ points in $Y$ and $m$ points in $\hat{y}$, then at each $X$ station, the difference approximation of equations (17) and (13), together with the interface conditions (14c) and (18c) can be written conveniently in matrix form as discussed by Bodonyi \& Duck ${ }^{12}$. The overall scheme is nominally second-order accurate in the grid spacings $\Delta X, \Delta Y$ and $\Delta \hat{Y}$. The resulting matrix equation is then solved using standard Gaussian elimination procedures and back substitution. 


\section{Numerical Results}

We first consider the effect of the strouhal number, $s_{o}$, on the interaction between the unsteady flow and the suction slot. To minimize any nonlinear effects, a small value of the wall suction velocity, $V_{W}=-0.1$, was chosen. The numerical solution was found for several values of $S_{0}$, ranging from 1.0 to 3.0 . In most cases 300 points were taken in the streamwise direction over the range $-5 \leq \mathrm{X} \leq$ $25(\Delta \mathrm{X}=0.10)$, although several cases were recomputed using 600 points $(\Delta X=0.05)$ in the streamwise direction as a check on the numerical accuracy of the solutions. In all cases the comparisons were very good, generally indistinguishable to graphical accuracy. To solve the lower-deck equations, 50 points were taken across the region over the range $0 \leq Y \leq 5$. Also, an additional 50 points were used in the upper-deck $\hat{Y}$ scaling over the range $0 \leq \hat{y} \leq 5$, to solve Laplace's equation for the pressure in the upper-deck region.

Using the finite-difference method of solution, convergence of the numerical computations was achieved when the absolute value of the difference in the displacement thickness, $R I\{a(X)\}$, where $R I\{\}$ denotes the real part of \{\} , between two successive iterates was less than $10^{-5}$ for all $x$. The number of iterations required for convergence was found to be sensitive to the value of $s_{0}$ under consideration and also to the initial guess taken for the disturbance profiles.

The disturbances produced by the interaction with the suction slot should ultimately decay sufficiently far downstream of the slot if the scaled freestream strouhal number is below its critical value, 
$S_{o_{\text {crit }}} \approx 2.29$. The real parts of the complex-valued disturbance pressure, $R I\left\{p_{O}(X)\right\}$, and wall shear, $R I\left\{\tau_{W}(X)\right\}=R I\left\{\partial u_{O} / \partial Y(X, 0)\right\}$, for a representative subcritical case, $s_{0}=1.5$, are presented in Figure 3. The decay in the disturbance amplitudes for both quantities is clearly evident for $\mathrm{X} \geq 5$. Similarly, the disturbances should amplify downstream of the surface distortion if $s_{0}$ is supercritical. The same $\mathrm{X}$ range was also considered for typical supercritical cases, $S_{0}=2.5$ and 3.0 . Figures 4 and 5 show the amplification of the disturbances in the streamwise direction for these cases. Note the change in scale between Figures 4 and 5 which indicates the rapid increase in the amplitudes of the disturbance profiles for supercritical disturbances.

We next consider the effect of increasing the magnitude of the wall suction parameter, $V_{W}$, for a strouhal number $s_{O}=2.5$. As can be seen in Figures $6-9$, the disturbance solutions for these cases have the same general shape as the previous solutions for $V_{W}=-0.1$. Note that when $V_{W} \leq-0.5$, the disturbance amplitudes for both the pressure and the wall shear decrease with downstream distance, at least for $X \leq 25$, in spite of the fact $S_{0}=2.5$ is supercritical and we would, therefore, expect the waves to be amplified. This effect clearly illustrates the damping effect that the appropriate amount of wall suction can have on the growth of the $\mathrm{T}-\mathrm{S}$ waves which are locally generated by the interaction of the free-stream disturbances with the suction slot. However, since $S_{0}$ is supercritical, the $T-S$ waves must eventually begin to amplify sufficiently far downstream. To see this effect, the case for $V_{W}=-0.75, S_{O}=2.5$ was recomputed 
for the $x$ range $-5 \leq x \leq 35$. The ultimate growth in the disturbance wall shear is evident in Figure 10 for $x \geq 30$.

To illustrate how our method can be used to simulate the concept of transition control, as mentioned in the Introduction, we consider the results for $S_{O}=3$, with $V_{W}=-0.10$. The results for the real part of the disturbance skin friction and pressure are shown in Figure 6. Note that these results were obtained using a fine X grid, with $\Delta \mathrm{X}=0.05$ (as opposed to the standard $\Delta \mathrm{X}=0.10$ grid); however, these results proved to be indistinguishable, on the scale shown, to those on the standard $x$ grid, in spite of the discontinuous normal velocity at $x= \pm 0.5$. These results also highlight, very clearly, the growing nature of the Tollmien-schlichting waves. In order to show how these waves may be effectively annihilated, we solved the related unsteady suction receptivity problem, i.e., (17) - (18), without the stokes solution, but with

$$
\begin{array}{ll}
\mathrm{v}_{0}=0 & \mathrm{y}=0,|\mathrm{x}|>\frac{1}{2}, \\
\mathrm{v}_{0}=1 & \mathrm{y}=0,|\mathrm{x}|<\frac{1}{2} .
\end{array}
$$

Results for this problem with $S_{0}=3$ are shown in Figure 11, and again show clearly the growing Tollmien-Schlichting wave pattern. Since the wavelength of these T-S waves is identical to those of the original receptivity problem, we are thus able to combine these two sets of results in such a way so as to eliminate the growing Tollmien-schlichting waves. If we denote the solution to the original problem as $u_{d}$, and that of the suction receptivity problem as $\mathbf{u}_{\mathrm{s}}$, we can then use superposition to write

$$
\mathbf{u}_{\mathrm{C}}=\mathbf{u}_{\mathrm{d}}+\beta \mathbf{u}_{\mathrm{S}} \text {. }
$$


Here the quantity $\beta$ was chosen such that

$$
p\left(X_{f}\right)=0 \text {, }
$$

viz.,

$$
\beta=-\mathrm{p}_{\mathrm{d}}\left(\mathrm{X}_{\mathrm{f}}\right) / \mathrm{p}_{\mathrm{S}}\left(\mathrm{X}_{\mathrm{f}}\right)
$$

where we chose $x_{f}=19.9$. Although this value is obviously arbitrary, experimentation did suggest this value was not crucial, confirming the robustness of the technique. This is also borne out by the results shown in Figure 12, clearly showing negligible values of the combined solution reasonably far downstream. 
v. Conclusions and Discussion

In this study we have investigated the interaction between a surface suction slot and a small amplitude unsteady disturbance to the freestream velocity.

Receptivity studies fall broadly into four distinct categories, depending on the degree of nonlinearity. The first, such as that studied by Goldstein ${ }^{5}$ involves a linear steady flow (the perturbation parameter being the surface distortion parameter) and a linear unsteady flow (the perturbation parameter being the amplitude of the unsteady distortion). The second category involves a nonlinear steady flow, together with a linear unsteady flow; this study and that of reference 8 fall into this class. The third category involves a fully nonlinear flow, with the steady flow being coupled, nonlinearly with the unsteadiness; the study of Duck $^{16}$ is of this class. However, the evidence is that many flows of this class, including incompressible flows, will ultimately culminate in a finite-time singularity. The fourth category may also exhibit such a breakdown, as studied in reference 16, where a single linearization is made, involving the surface distortion parameter.

In this paper we have shown how, at least in principle, T-S waves may be effectively cancelled out by a second unsteady mechanism, carefully chosen. As noted in the Introduction, such a technique has obvious practical applications in the field of laminar flow control. 


\section{Acknowledgement}

The authors are grateful to the ohio supercomputer Center for making computer time available on the Cray $\mathrm{Y}-\mathrm{MP} / 864$ computer during this research program. Additional computer time was provided by S.E.R.C. under grant Number GR/E/25702, and by the University of Manchester. This research was supported by the National Aeronautics and Space Administration under NASA Contract No. NAS1-18605 while the authors were in residence at the Institute for Computer Applications in Science and Engineering (ICASE), NASA Langley Research Center, Hampton, VA. RJB would like to acknowledge the Institute for Computational Mechanics in Propulsion (ICOMP) program at the NASA Lewis Research Center, Cleveland, $\mathrm{OH}$, where a portion of this research was carried out. 


\section{REFERENCES}

1. Reshotko, E. 1976, In Ann. Rev. Fluid Mech., V. 8, 311.

2. Murdock, J.W. 1980, Proc. Roy. Soc. Lond., A372, 517.

3. Goldstein, M.E. 1983, J. Fluid Mech., V. 127, 59.

4. Goldstein, M.E. 1984, J. Fluid Mech., V. 145, 71.

5. Goldstein, M.E. 1985, J. Fluid Mech., V. 154, 509.

6. Goldstein, M.E., Sockol, P.M. \& Sanz, J. 1983, J. Fluid Mech., V. 129, 443 .

7. Goldstein, M.E., Leib, S.J. \& Cowley, S.J. 1987, J. Fluid Mech., v. 181,485 .

8. Bodonyi, R.J., Welch, W.J.C., Duck, P.W. \& Tadjfar, M. 1989, J. Fluid Mech., V. 209, 285.

9. Liepman, H.W., Brown, G.L. \& Nosenchuck, D.M. 1982, J. Fluid Mech., V. 118, 187.

10. Liepman, H.W. \& Nosenchuck, D.M. 1982, J. Fluid Mech., V. 118, 201.

11. Smith, F.T. 1979, Proc. RoY. Soc. Lond., A368, 573.

12. Bodonyi, R.J. \& Duck, P.W. 1988, Computers \& Fluids, V. 16, 279.

13. Duck, P.W. 1985, J. Fluid Mech., V. 160, 465.

14. Smith, F.T. \& Bodonyi, R.J. 1985, Aeronautical J., V. 89, 205.

15. Bodonyi, R.J., Smith, F.T. \& Gajjar, J. 1983, IMA J. Appl. Math., V. 30,1 .

16. Duck, P.W. 1988, J. Fluid Mech., V.147, 254. . 


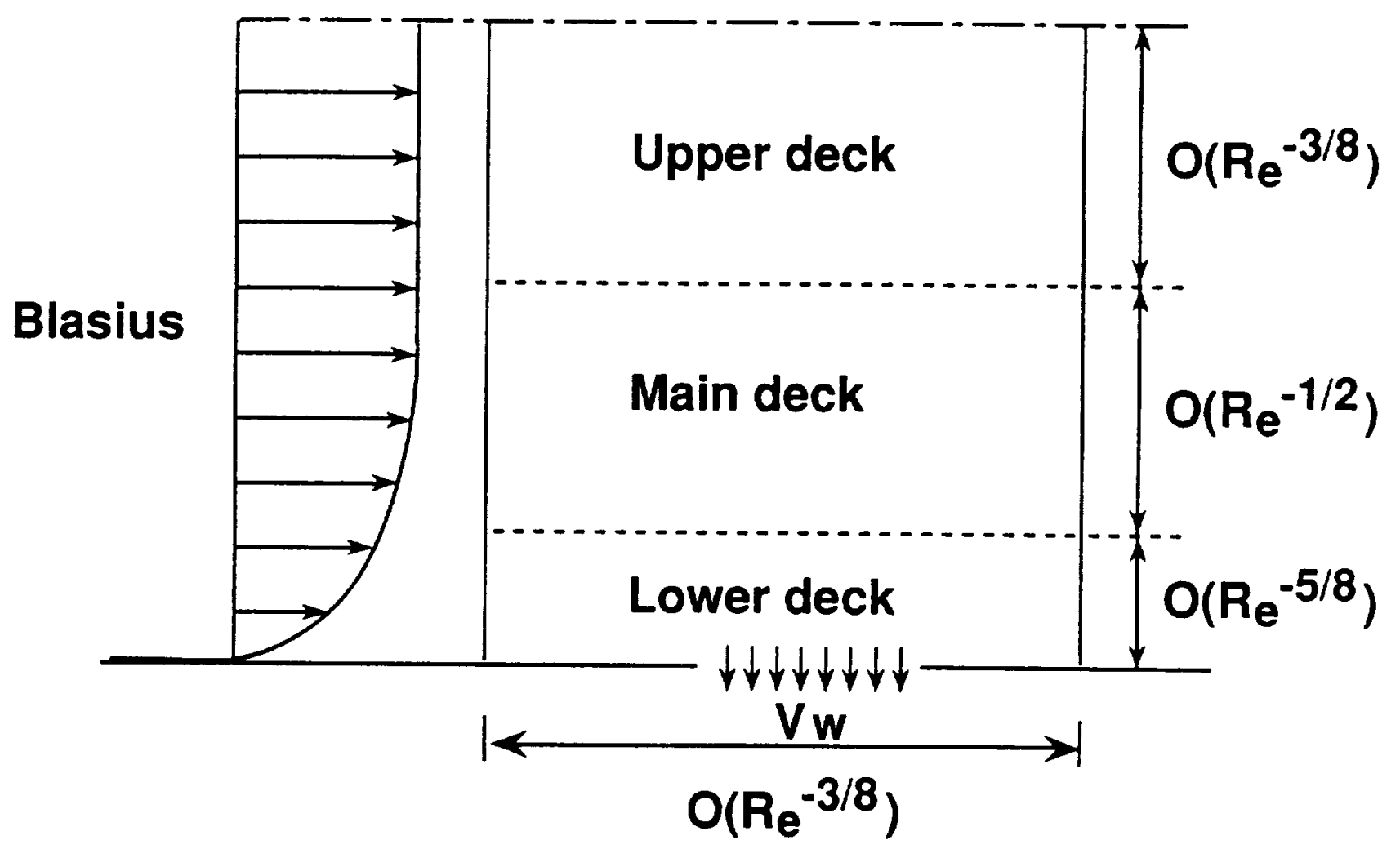

Figure 1. Schematic of the triple-deck flow structure. 


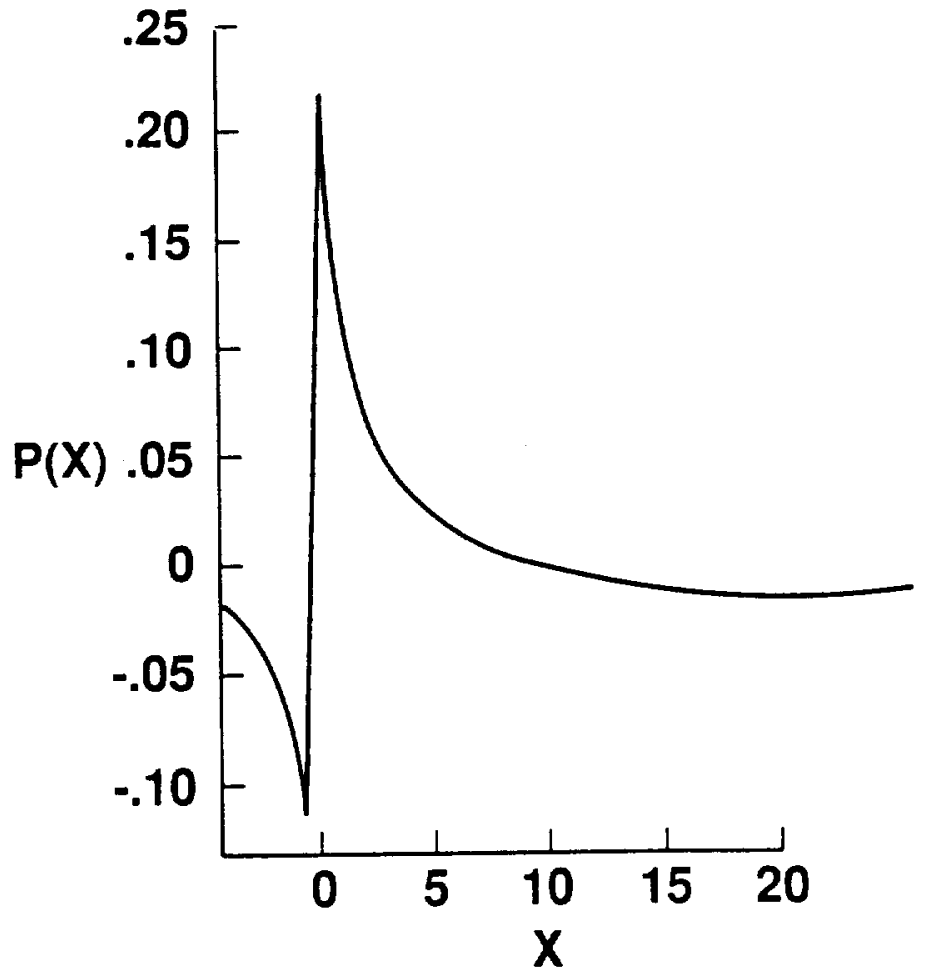

Figure $2 a$.

Pressure distribution for steady flow with $h=5$.

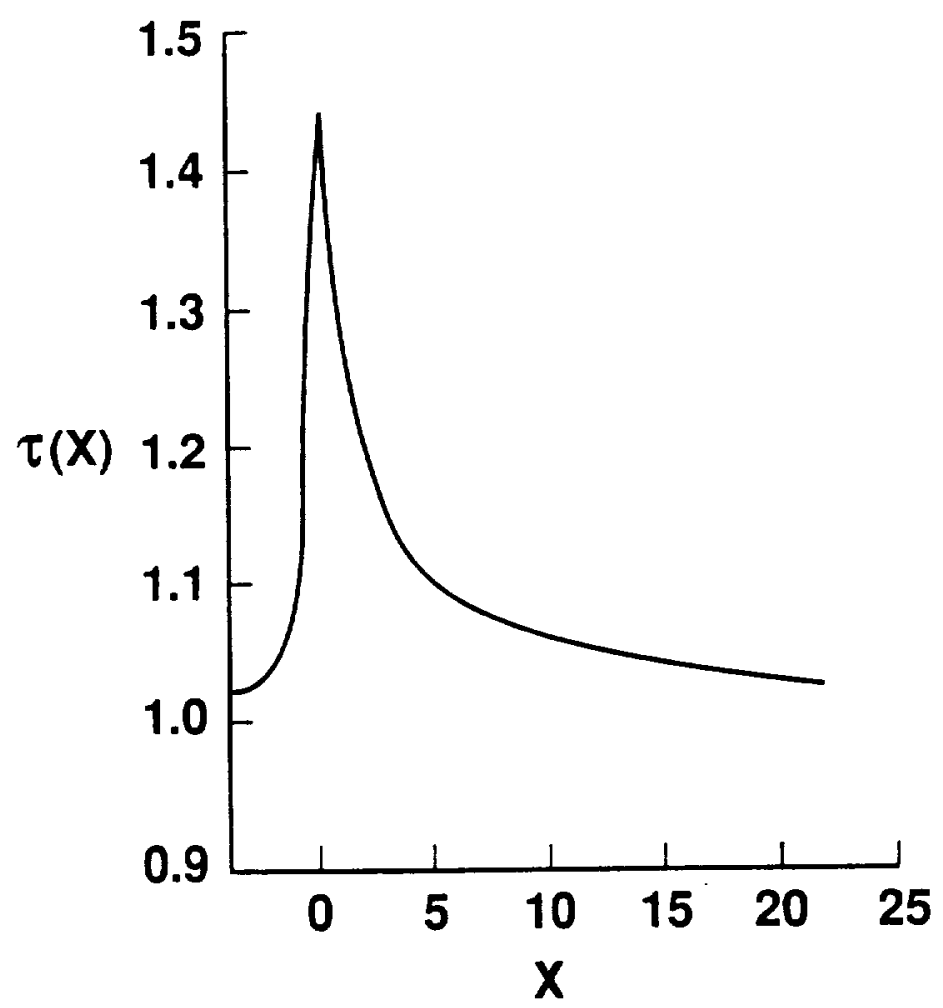

Figure 2b.

Wall shear distribution for steady flow with $h=5$. 


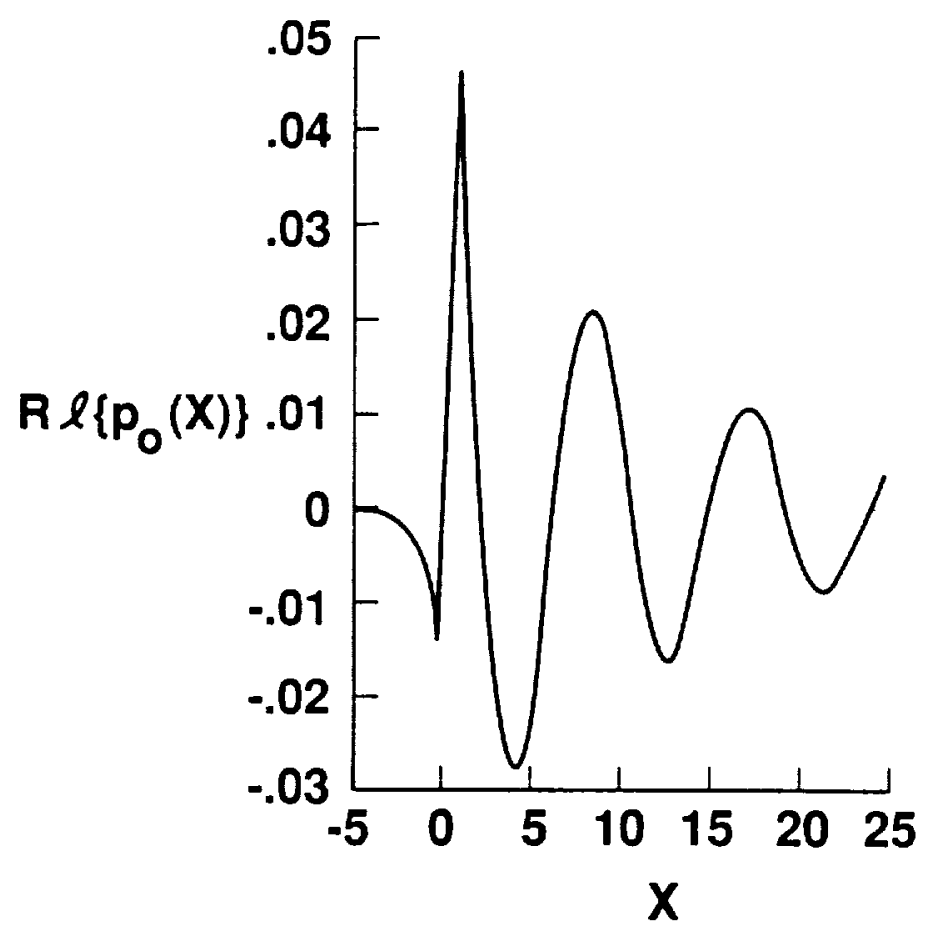

Figure 3a. Disturbance pressure for $s_{0}=1.5, V_{w}=-0.10$.

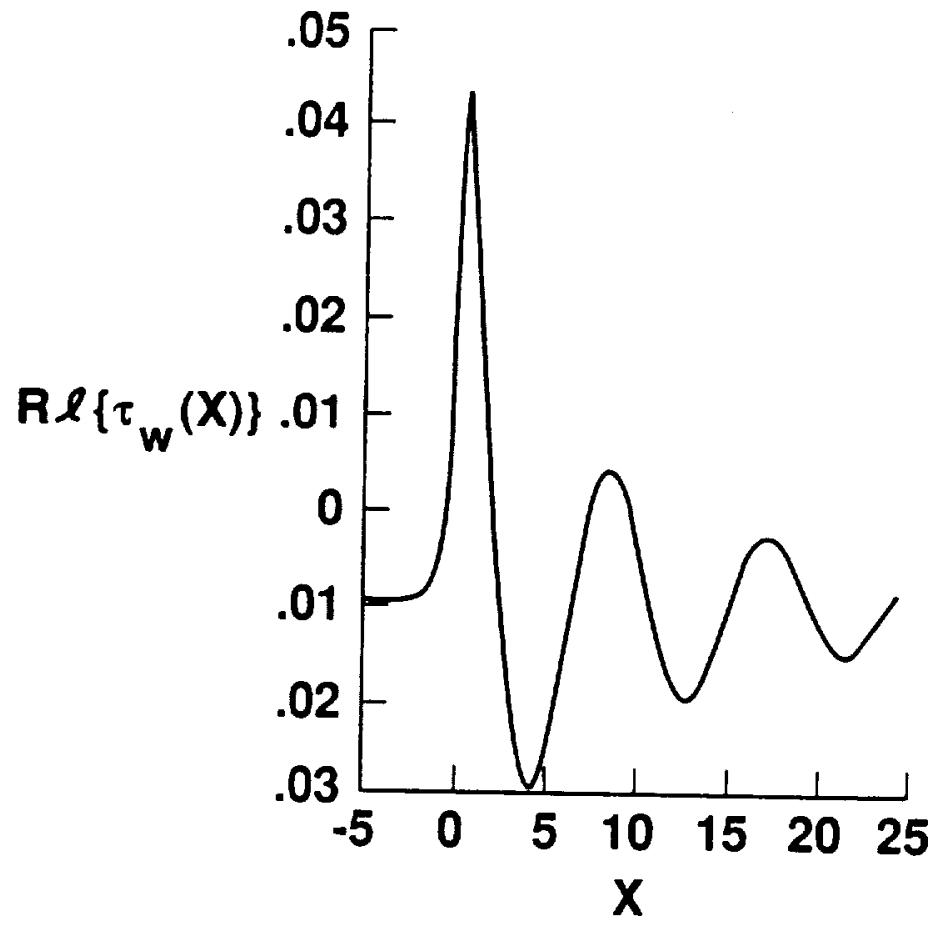

Figure 3b. Disturbance wall shear for $s_{0}=1.5, V_{W}=-0.10$. 


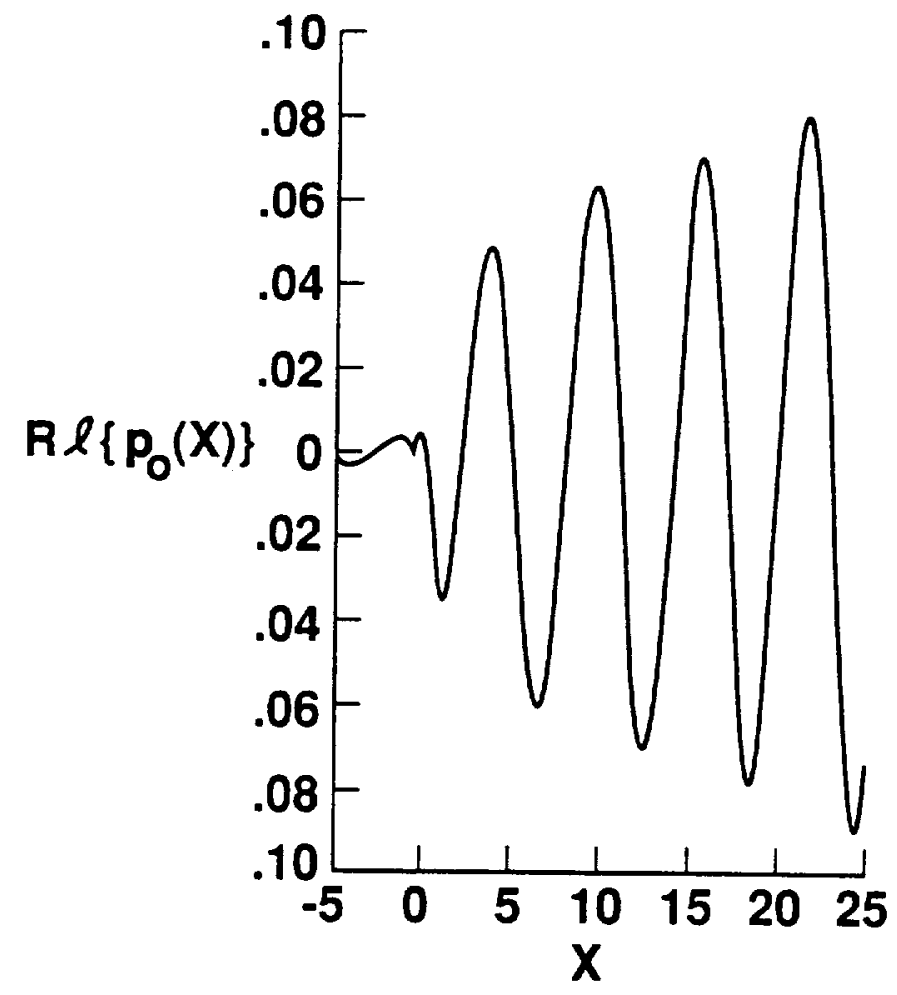

Figure 4a. Disturbance pressure for $s_{0}=2.5, v_{w}=-0.10$.

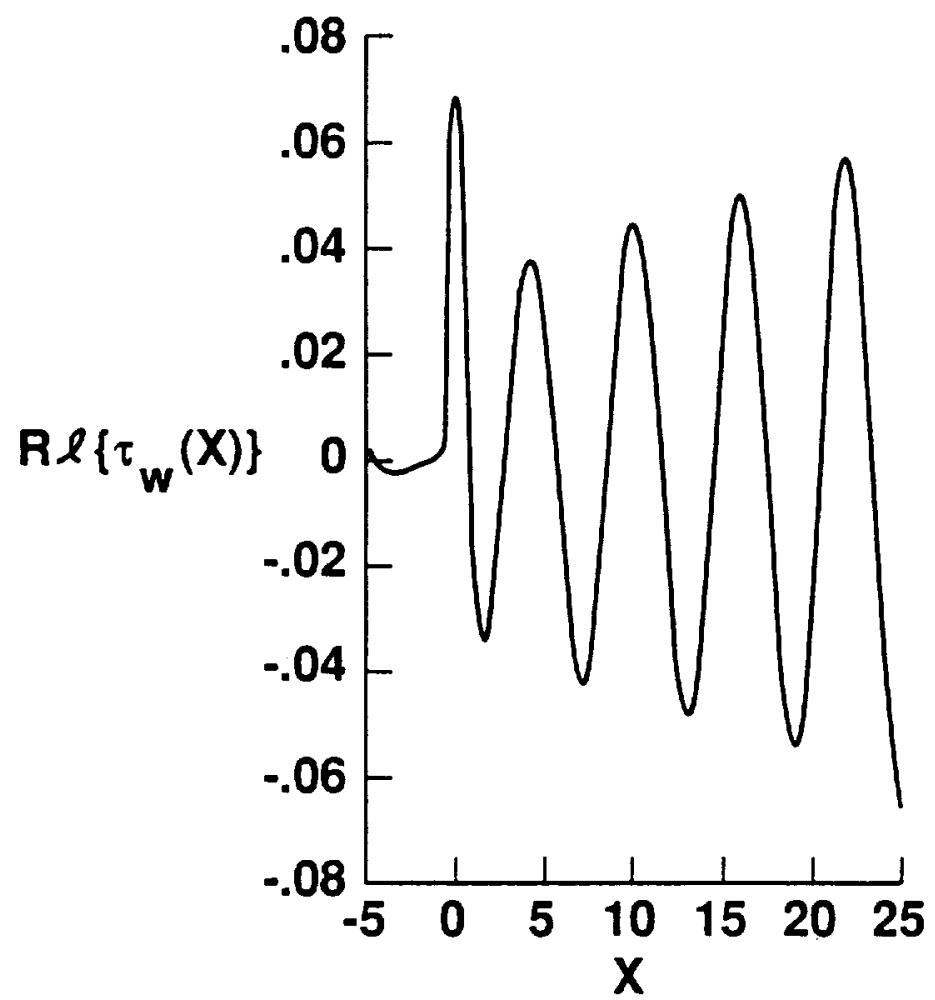

Figure 4b. Disturbance wall shear for $s_{0}=2.5, v_{W}=-0.10$. 


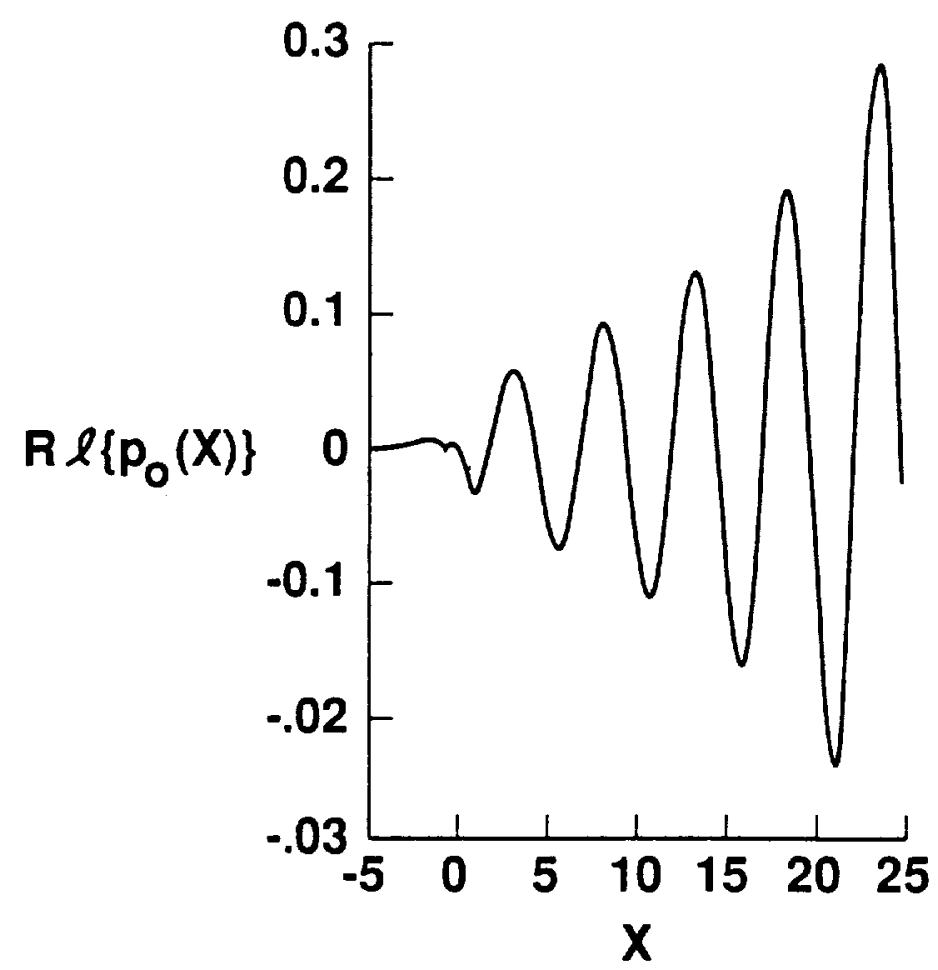

Figure 5a. Disturbance pressure for $s_{0}=3, v_{w}=-0.10$.

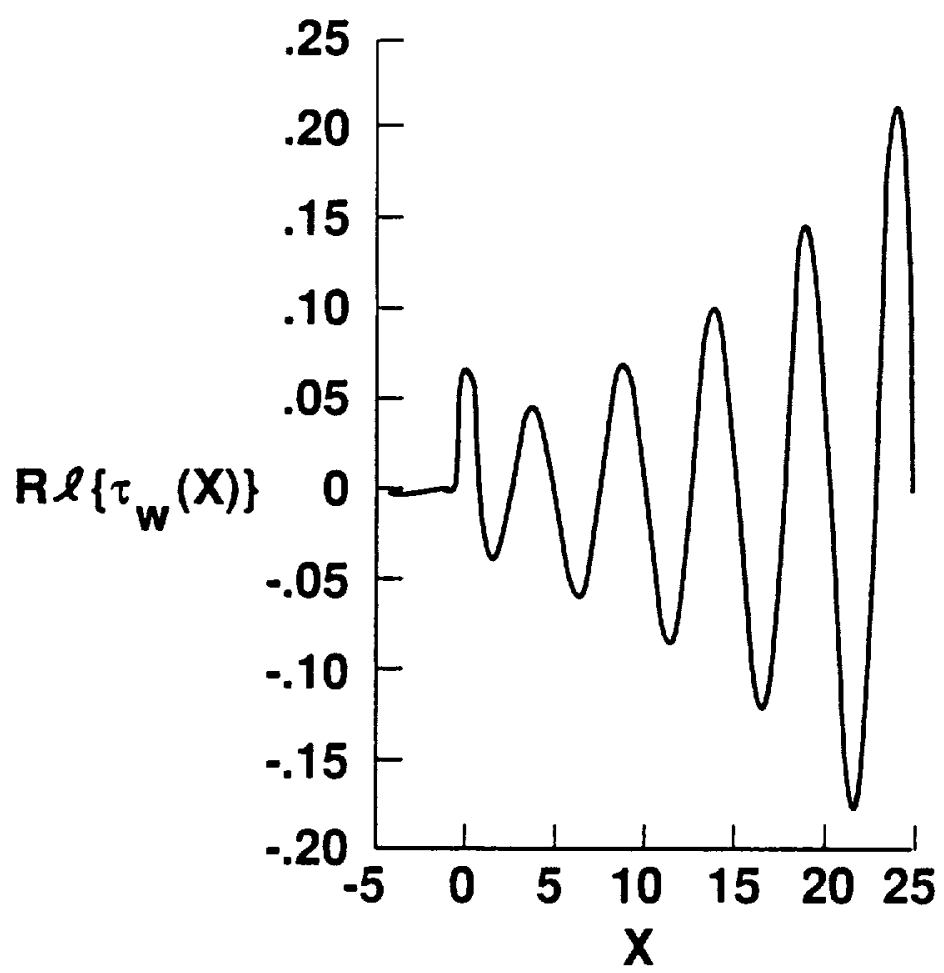

Figure 5b. Disturbance wall shear for $s_{0}=3, v_{w}=-0.10$. 


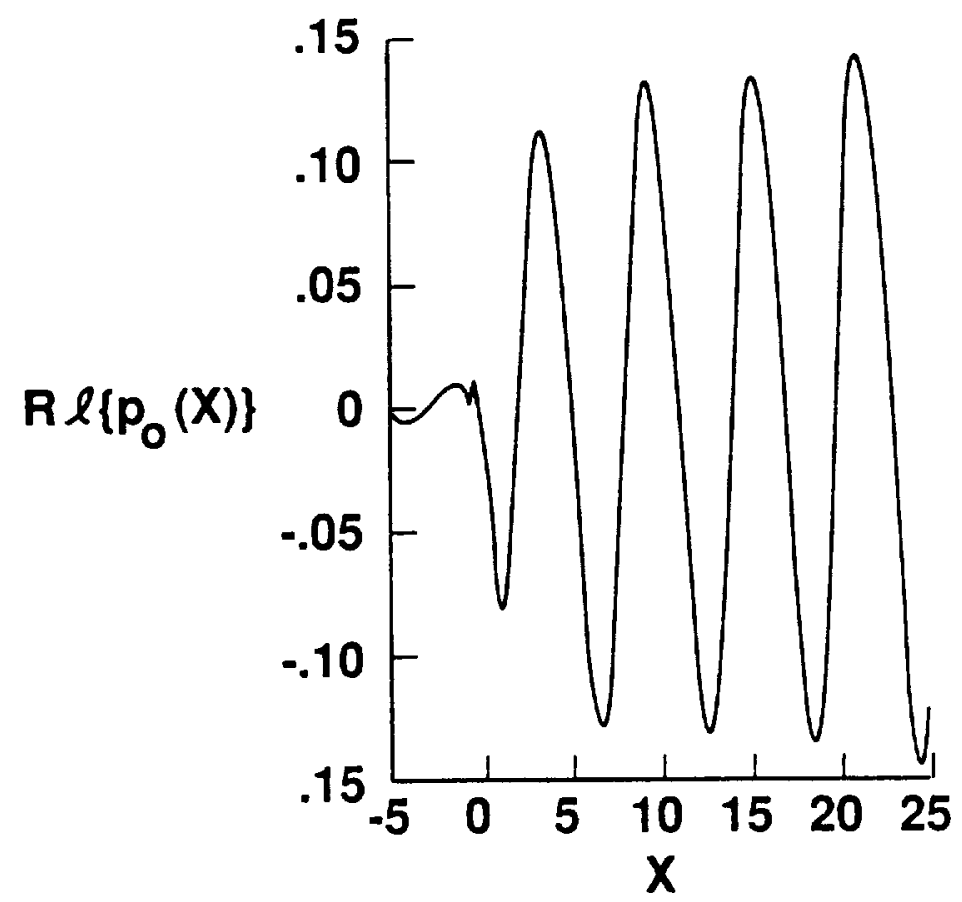

Figure 6a. Disturbance pressure for $S_{0}=2.5, V_{W}=-0.25$.

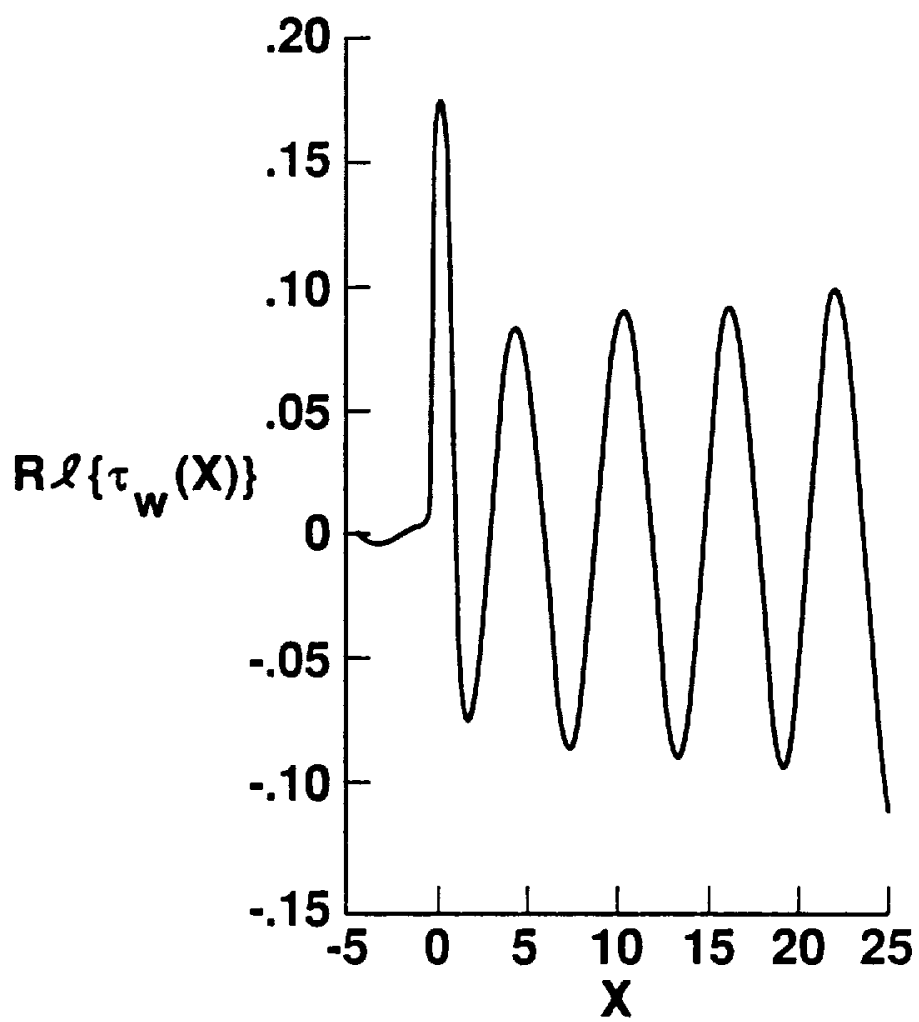

Figure 6b. Disturbance wall shear for $S_{0}=2.5, V_{w}=-0.25$. 


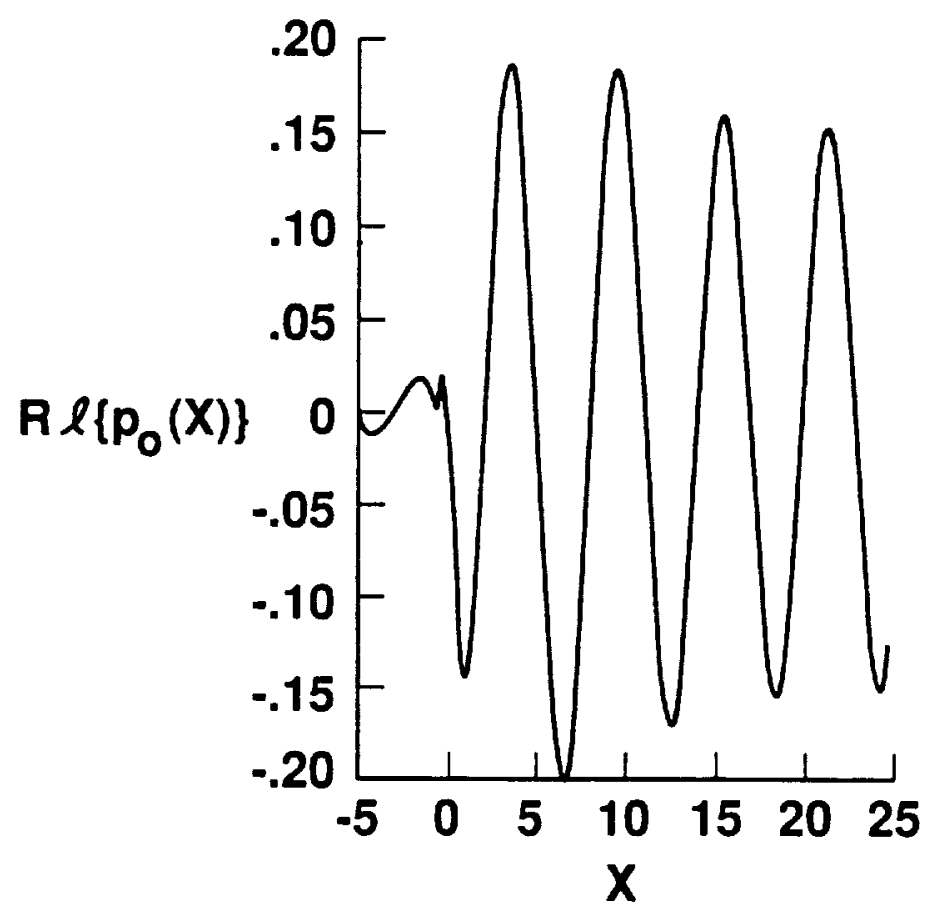

Figure 7a. Disturbance pressure for $s_{0}=2.5, V_{W}=-0.50$.

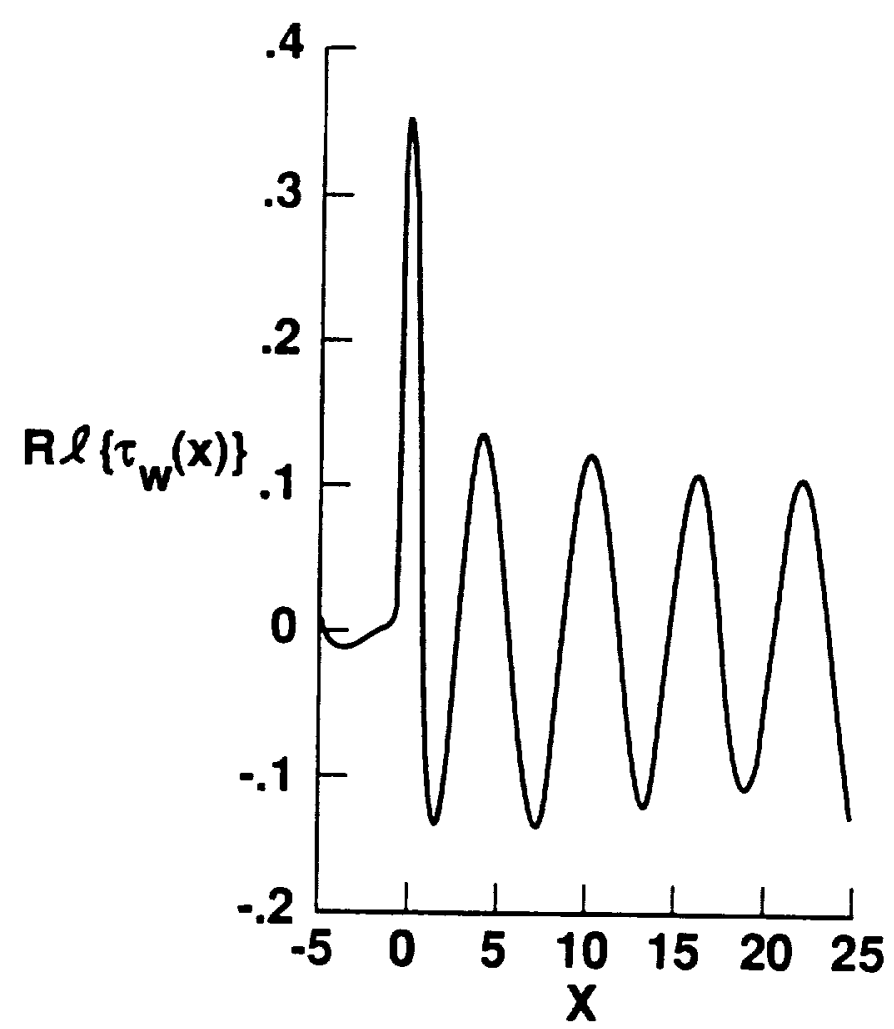

Figure 7b. Disturbance wall shear for $s_{0}=2.5, V_{W}=-0.50$. 


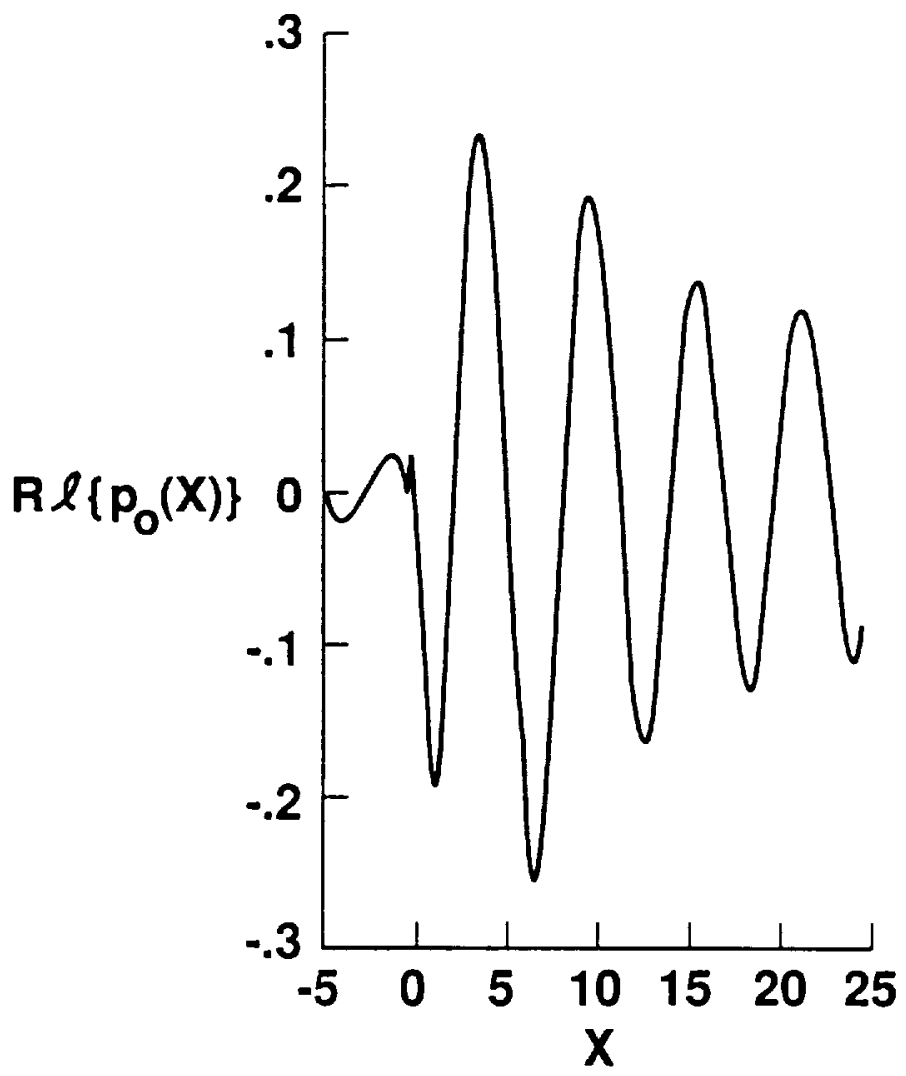

Figure 8a. Disturbance pressure for $S_{0}=2.5, v_{W}=-0.75$.

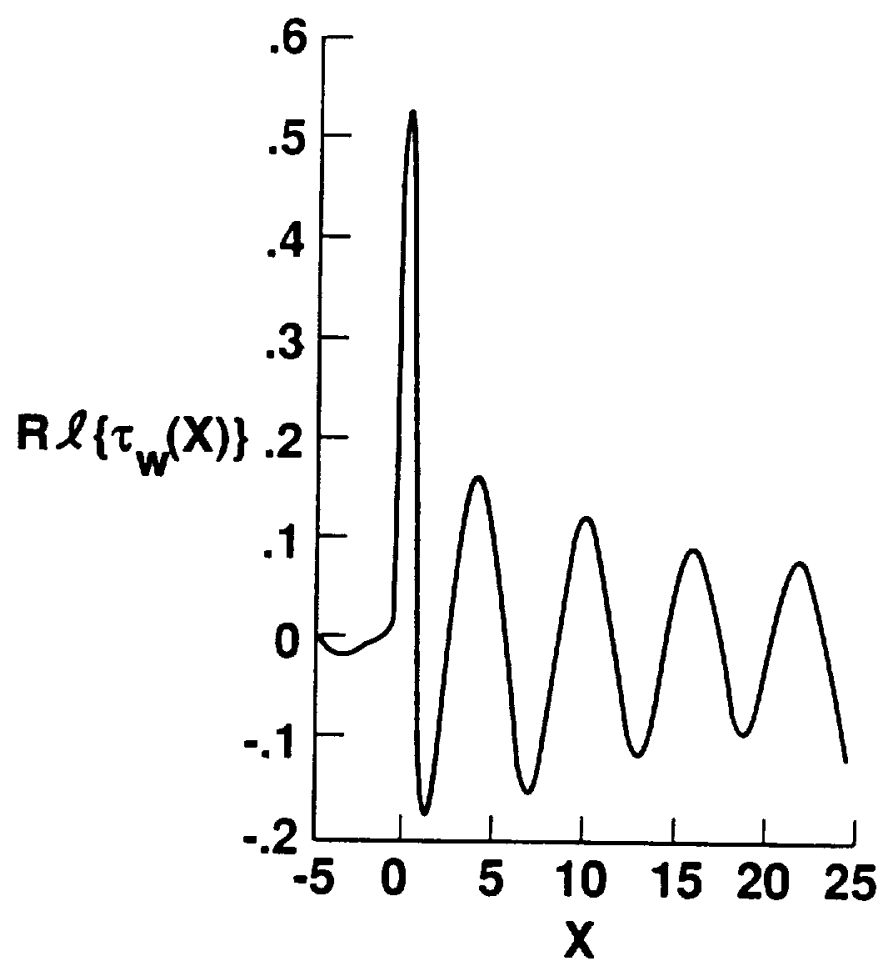

Figure 8b. Disturbance wall shear for $S_{0}=2.5, V_{w}=-0.75$. 


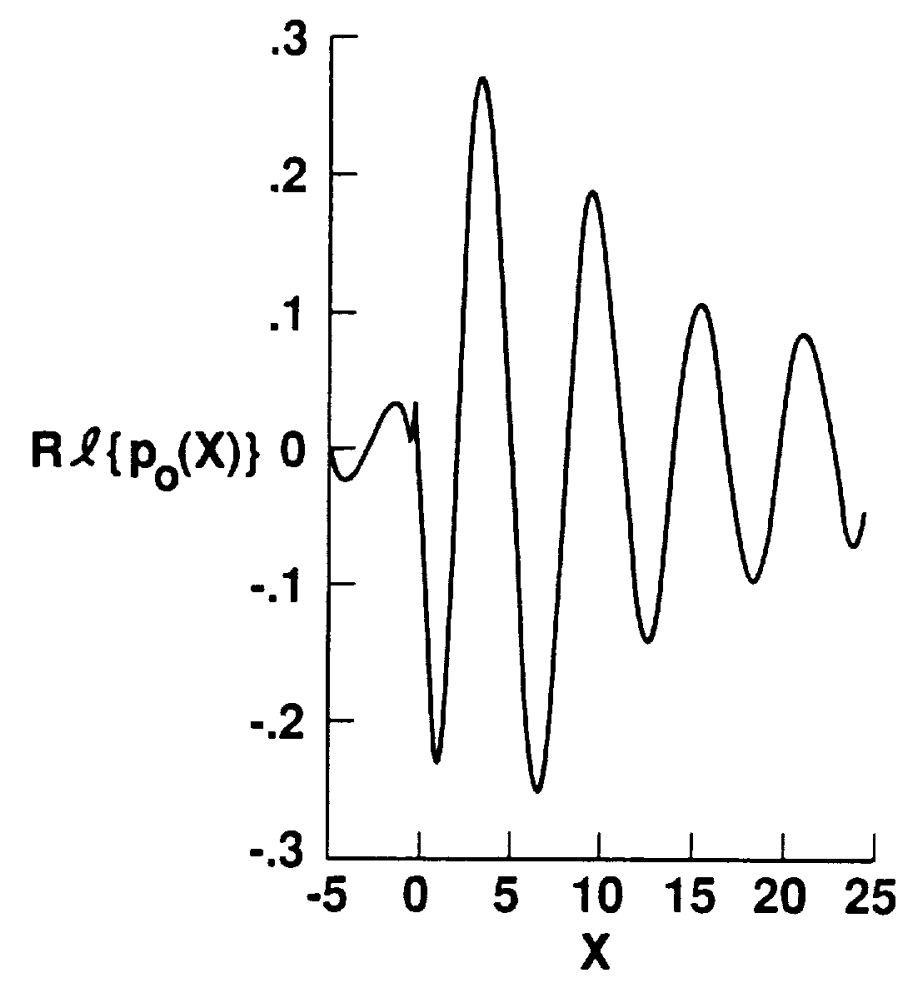

Figure 9 . Disturbance pressure for $S_{0}=2.5, V_{W}=-1.0$.

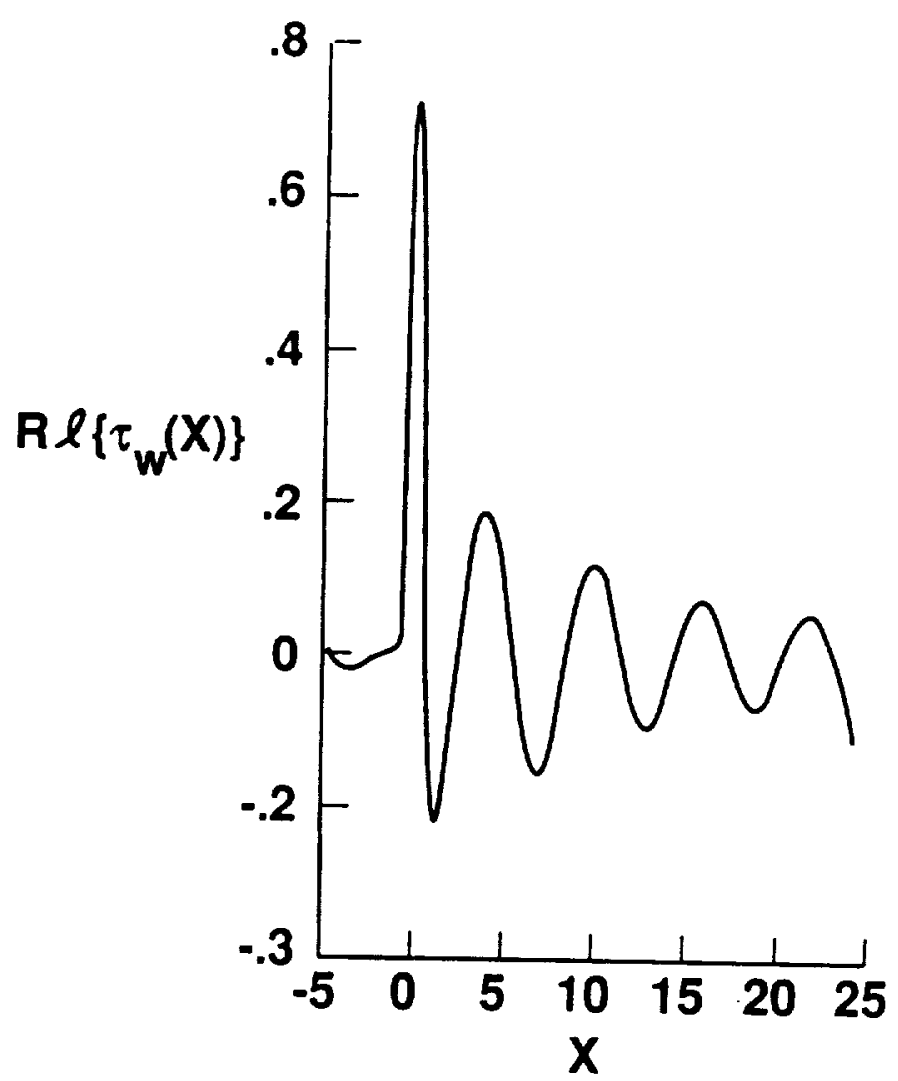

Figure 9b. Disturbance wall shear for $s_{0}=2.5, v_{w}=-1.0$. 


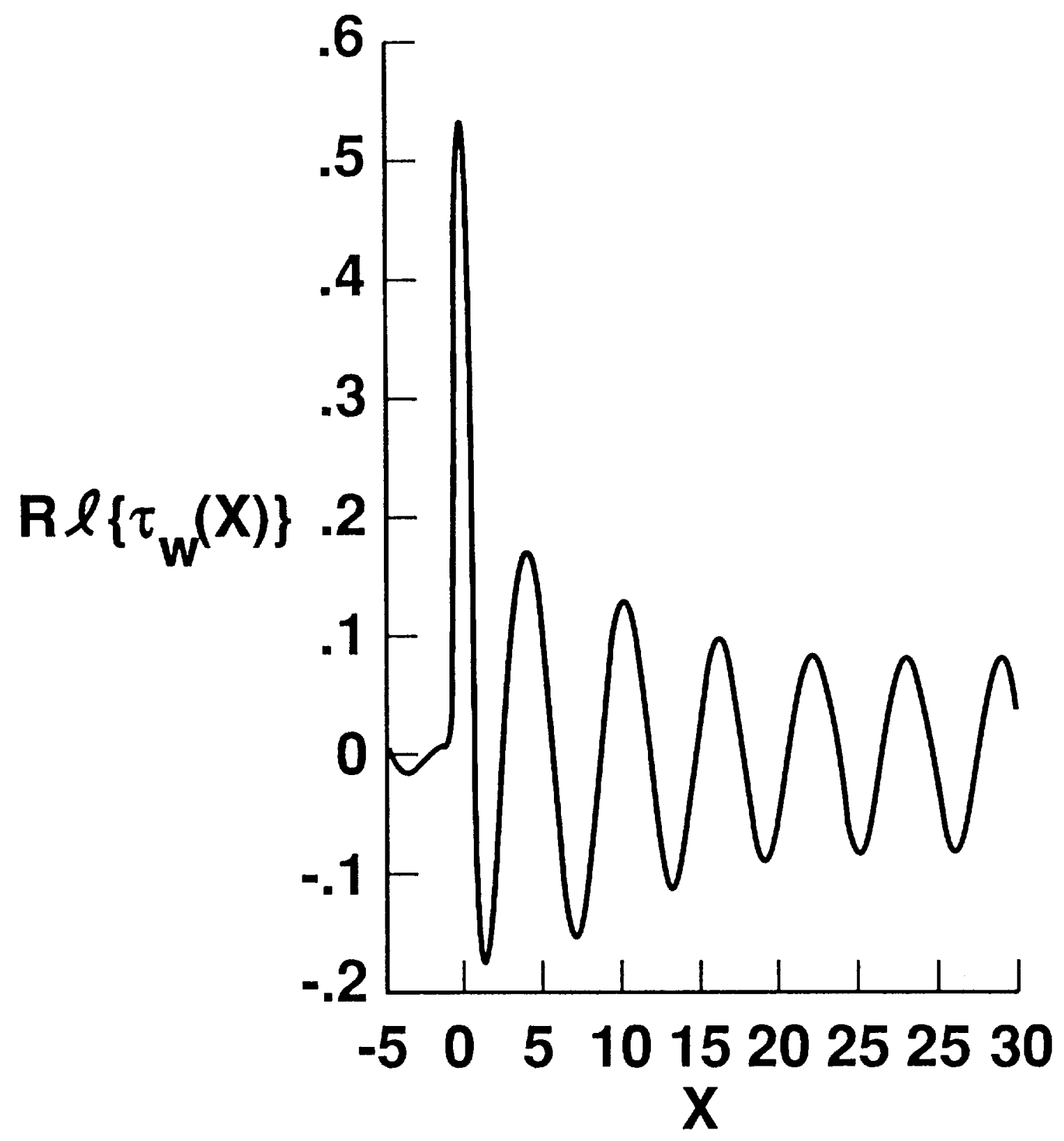

Figure 10. Disturbance wall shear for $S_{0}=2.5, V_{W}=-0.75$, and $-5 \leq \mathrm{X} \leq 35$. 


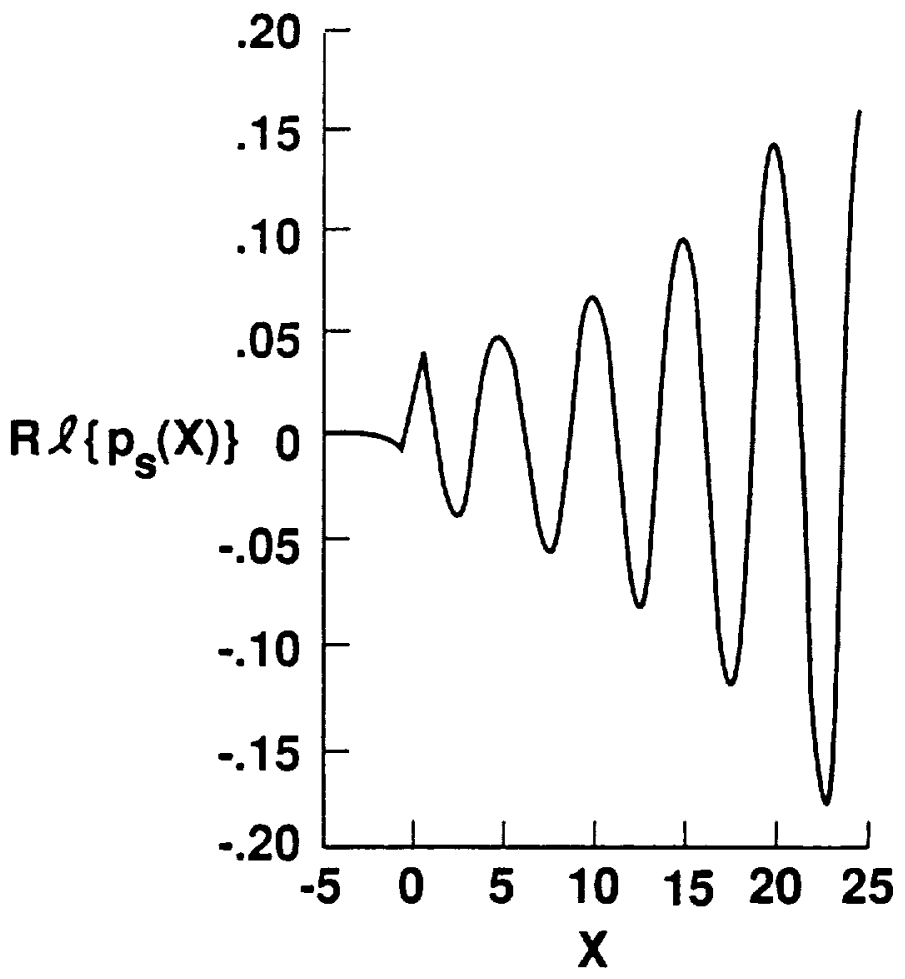

Figure 11a. Disturbance pressure for the suction receptivity problem $\left(u_{S}\right)$ for $S_{0}=3.0, V_{W}=-0.10$.

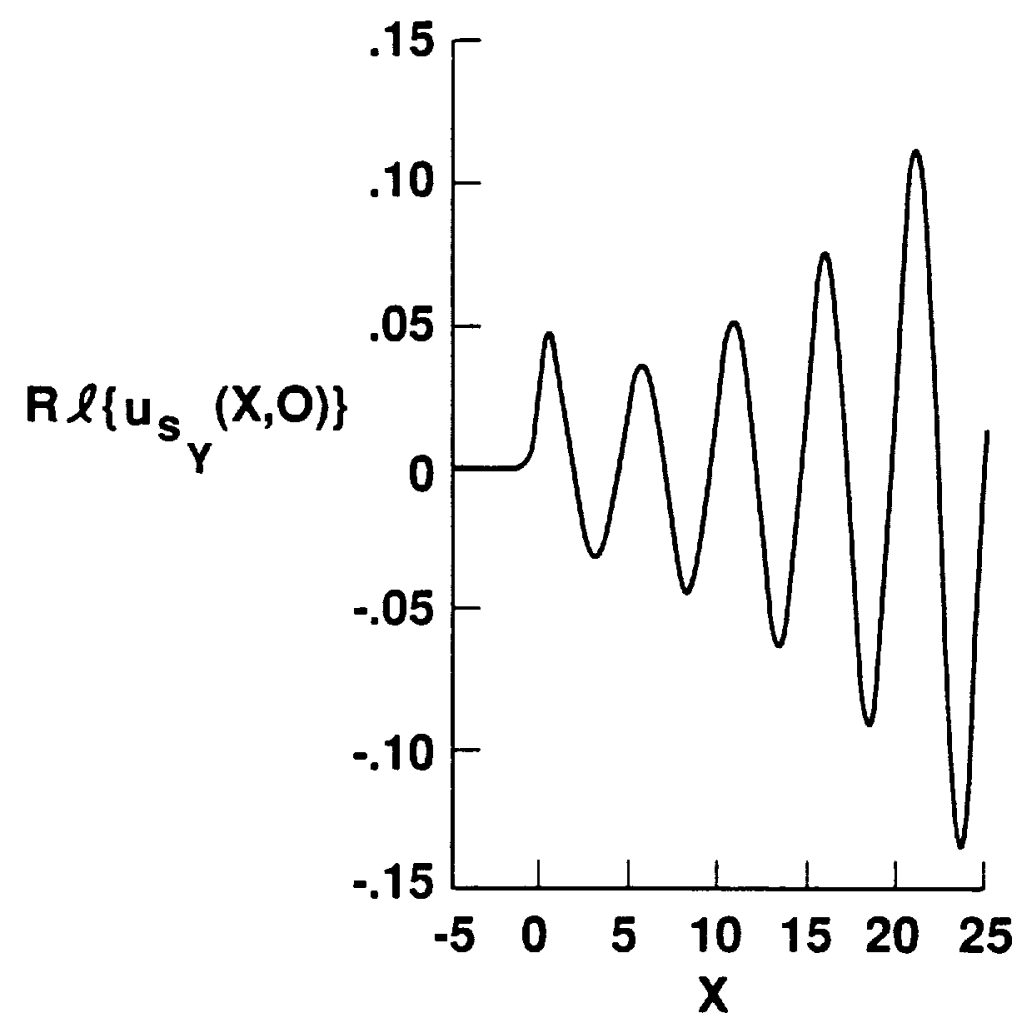

Figure 11b. Disturbance wall shear for the suction receptivity problem $\left(u_{s}\right)$ for $s_{0}=3.0, v_{w}=-0.10$. 


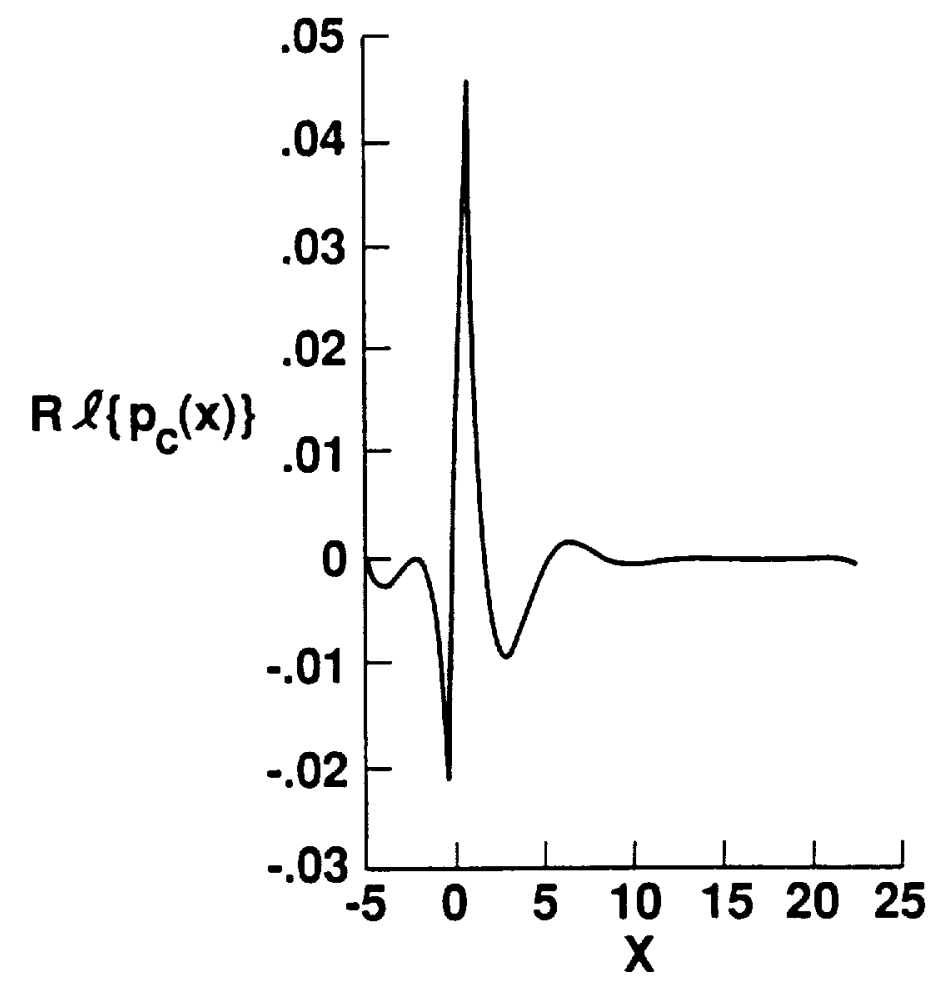

Figure 12a. Disturbance pressure for the combined receptivity problem $\left(u_{C}\right)$ for $S_{0}=3.0, v_{w}=-0.10$.

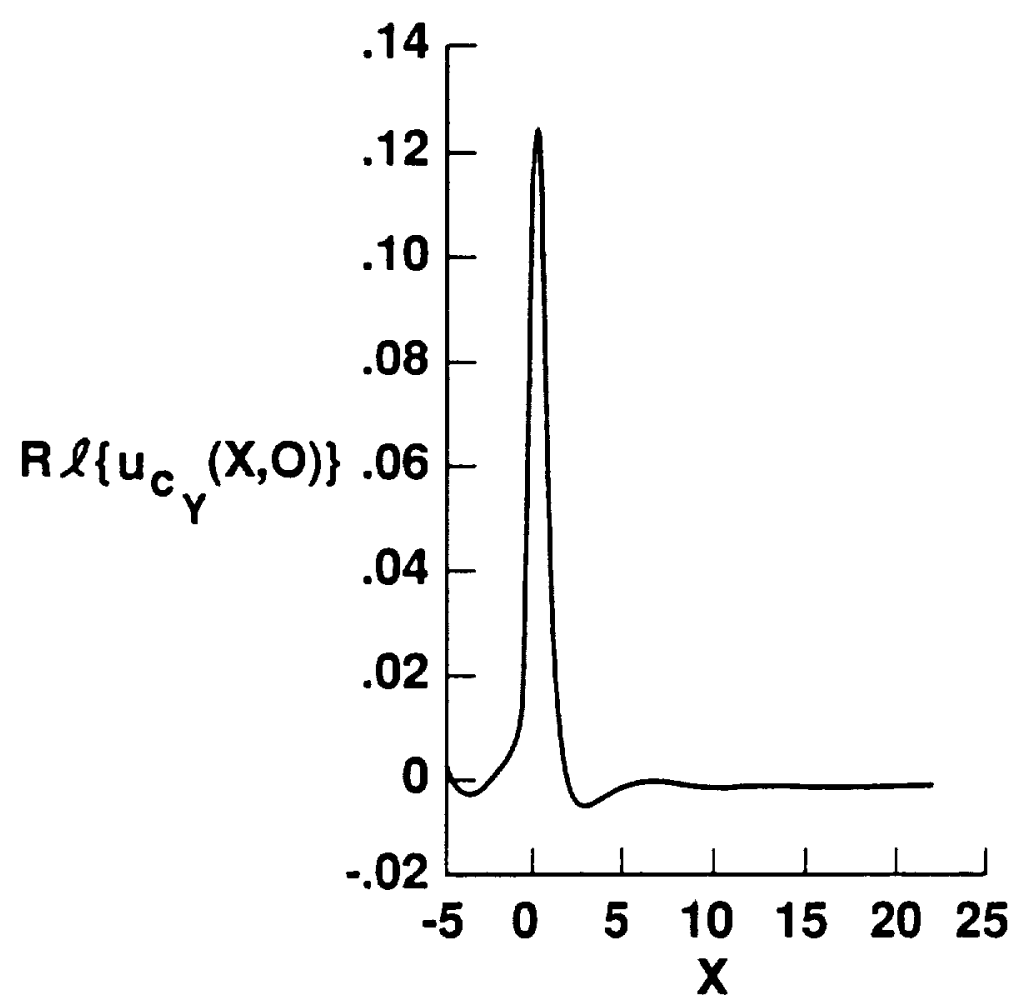

Figure 12b. Disturbance wall shear for the combined receptivity problem $\left(u_{c}\right)$ for $s_{0}=3.0, v_{w}=-0.10$. 



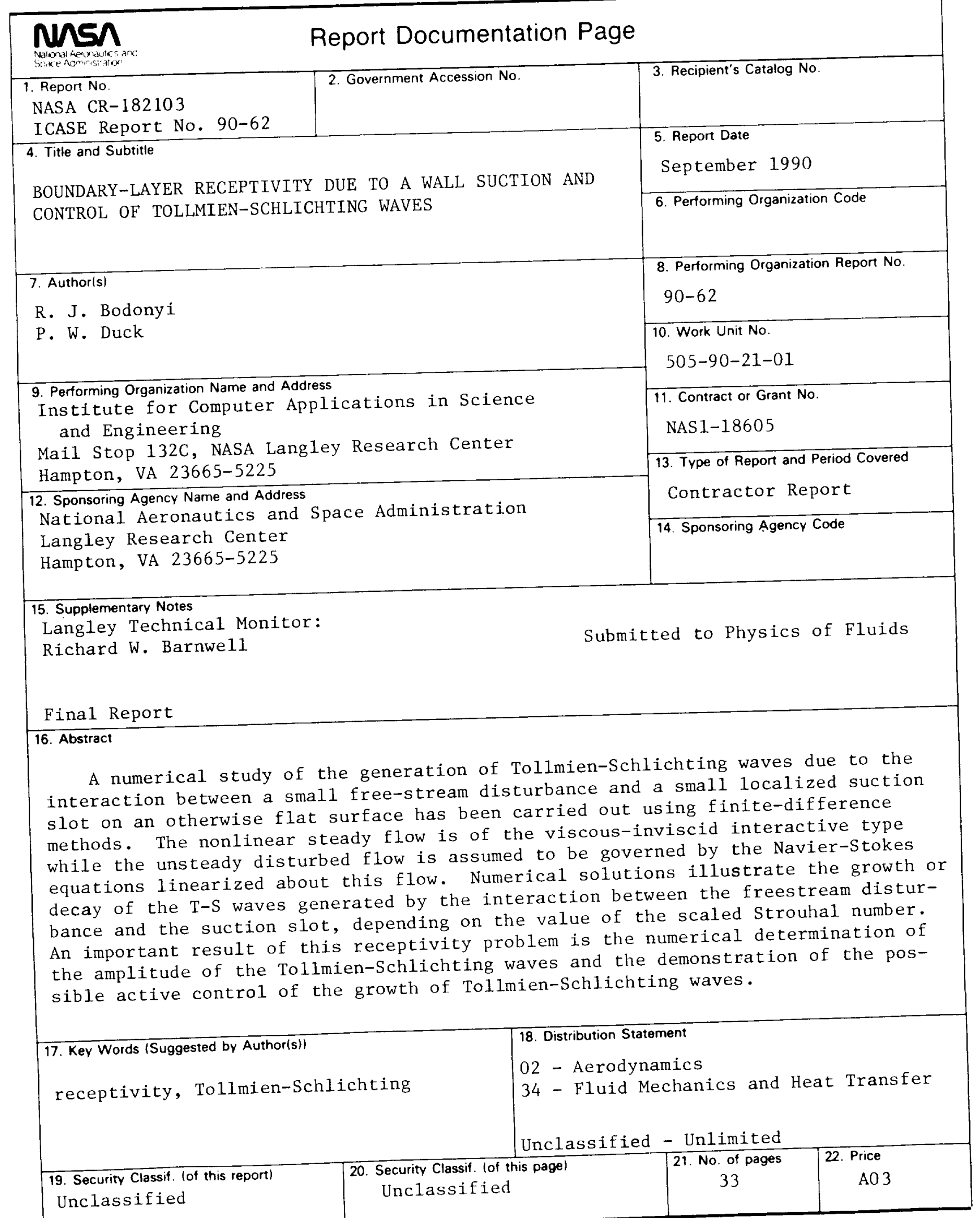



